\title{
3 Research Square \\ Platelet Phagocytosis by Neutrophils, Platelets Lack of Granules and Giant Platelets Result in Pseudothrombocytopenia
}

\section{Ya Zhu}

the first people's hospital of tianmen city

Junyang Zhou

Xuzhou Medical University https://orcid.org/0000-0002-6999-0781

Li Sun

xiangyang vocational and technical college

Feibo Guo

the first people's hospital of tianmen city

Yan Ding

Hubei University of Medicine

Mingchao Zhu (D 645249756@qq.com)

the first people's hospital of tianmen city

\section{Case Report}

Keywords: tuberculosis, 2HRZE/4HR, platelet phagocytosis, pseudothrombocytopenia

Posted Date: October 13th, 2021

DOl: https://doi.org/10.21203/rs.3.rs-923994/v1

License: (9) (i) This work is licensed under a Creative Commons Attribution 4.0 International License.

Read Full License 


\section{Abstract}

Pseudothrombocytopenia (PTCP) is a condition in which the decreased platelet count does not agree with the clinical status of the patient, can lead to misdiagnose, unnecessary tests, unnecessary treatment. The present case describes a 73-year-old man suffered with pulmonary tuberculosis, treated with antituberculosis therapy (isoniazid, rifampicin, pyrazinamide, ethambutol, 2HRZE/4HR). One month later, the patient had a significant decrease in platelets (101 to $\left.56 \times 10^{9} / \mathrm{L}\right)$. Peripheral blood smear showed that $28 \%$ platelets were phagocytosis by neutrophils, $26 \%$ platelets were lack of granules and $6 \%$ platelets' volume increased significantly. When the anticoagulant was changed from EDTA to sodium citrate, there was no change in the above phenomenon. By manual count, the value of platelets was $113 \times 10^{9} / \mathrm{L}$. After the completion of anti-tuberculosis therapy, platelet morphology gradually returned to normal. HRZE treatment may cause platelet morphology abnormal, resulting in PTCP. In such cases, we should regularly review the peripheral blood smear to ensure the accuracy of the results and avoid unnecessary examination and treatment. The emergence of PTCP may does not mean the presence of specific disorders.

\section{Background}

Thrombocytopenia can have several causes, including platelet clumping or formation of platelet rosettes around neutrophils, the use of certain drugs. When the decreased platelet count does not agree with the clinical status of the patient, incorrect measurements of platelet may lead PTCP, then cause the mistake of diagnostic and treatment. The knowledge of PTCP is important for the accuracy of a clinical assessment and for avoiding unnecessary treatment [1]. PTCP is usually caused by improper blood collection, thylenediaminetetraacetic acid dependent pseudothrombocytopenia (EDTA-PTCP), platelet volume increases or decreases [2,3]. The mechanism is not clearly defined so far. Aggregation is the most common phenomenon while platelet phagocytosis is the rarest one in PTCP $[4,5]$.

This little known in vitro phenomenon still under recognized may lead to misdiagnosis of thrombocytopenia, overtreatment, and further, even invasive, unnecessary testing. The present case describes a case platelet phagocytosis by neutrophils, platelet lack of granules and giant platelet result in PTCP. We regularly examined the peripheral blood cell morphology of our patient, there was no platelet satellite and EDTA-dependent platelet phenomena. Anti-tuberculosis treatment may cause platelet morphology abnormal, resulting in PTCP. In such cases, we should regularly review the peripheral blood smear to ensure the accuracy of the results and avoid unnecessary examination and treatment

\section{Case Presentation}

A 73-year-old man presented to our hospital with chest discomfort, cough, no fever. The patient has a 30 year history of chronic hepatitis $B$, his liver function was normally. He has no history of hypertension, diabetes or heart disease, drug allergy, respiratory diseases, Corona Virus Disease 2019 (COVID-19). The patient had a long history of smoking and drinking. 
The patient's platelet was $101 \times 10^{9} / \mathrm{L}$ on admission, peripheral blood smear showed no obvious change in morphology. Routine laboratory tests showed that there was no obvious abnormality in white blood cell (WBC), liver and kidney function, or coagulation function. Chest computed tomography (CT) showed bilateral lung infection, emphysema, pulmonary tuberculosis and cavitation were considered. On physical examination, breathing sounds in both lungs were rough, and a few moist crackles could be heard in the left upper lung. Mycobacterium tuberculosis rifampicin resistance gene test and serum tuberculosis antibody test were positive. Combined with the patient's symptoms, signs, CT scan and laboratory tests, he was diagnosed with tuberculosis. Subsequently, he accepted anti-tuberculosis therapy (isoniazid, rifampicin, pyrazinamide, ethambutol, 2HRZE/4HR) on the daily dose recommended by the World Health Organization tuberculosis guidelines.

One month later, laboratory tests showed that there was no obvious abnormality in WBC, liver and kidney function, or coagulation function, but there was a significant decrease in platelets $\left(56 \times 10^{9} / \mathrm{L}\right.$ reference range: $125-350 \times 10^{9} / \mathrm{L}$ ) (Table1). Peripheral blood smear showed that $28 \%$ platelets were phagocytosis by neutrophils, $26 \%$ platelets were lack of granules and $6 \%$ platelets' volume increased significantly, no platelet satellite was observed, other cells presented no morphological abnormalities (Table1 and Fig. 1). When the anticoagulant was changed from EDTA to sodium citrate, there was no change in the above phenomenon. By manual count, the value of platelets was $136 \times 10^{9} / \mathrm{L}$. The patient did not have any bleeding tendency. Therefore, we believe that the patient developed PTCP and did not need to undergo bone marrow puncture and platelet enhancement therapy. In subsequent testing, platelet phagocytosis, platelet lack of granules and giant platelet, all these situation existed all the time(Table1).Three months after the completion of antituberculous therapy, platelet morphology gradually returned to normal(Table1).

Table 1

Laboratory tests results of the patient. platelet (PLT), reference range: $125-350 \times 10^{9} / \mathrm{L}$.

\begin{tabular}{|lcccccc|}
\hline PLT & $\begin{array}{l}\text { Jan- } \\
\mathbf{2 7}\end{array}$ & Mar-2 & Mar-10 & May-19 & Jun-14 & Sep-30 \\
\hline Instrument count $\left(\times 10^{9} / \mathrm{L}\right)$ & 89 & 56 & 58 & 74 & 46 & 132 \\
\hline Manual count $\left(\times 10^{9} / \mathrm{L}\right)$ & 101 & 136 & 121 & 109 & 93 & 136 \\
\hline $\begin{array}{l}\text { Platelet phagocytosis by neutrophils } \\
(\%)\end{array}$ & 0 & 28 & 22 & 20 & 20 & 0 \\
\hline Platelet lack of granules $(\%)$ & 0 & 26 & 20 & 20 & 18 & 0 \\
\hline Giant platelet $(\%)$ & 0 & 6 & 4 & 6 & 11 & 1 \\
\hline Total rate of abnormal platelets $(\%)$ & 0 & 60 & 46 & 46 & 49 & 1 \\
\hline$-:$ none. Detecting instrument: Japan, Sysmex, XN9000. & & & & \\
\hline
\end{tabular}




\section{Discussion}

Platelets play an important role in the body, such as regulators of hemostasis and thrombosis. In pathological conditions, platelets are essential for formation of occlusive thrombus formation .Platelets have also been shown to play an important role in innate immunity as well as regulation of tumor growth and extravasations in the vessel[6]. Once the number or function of platelets is abnormal, further testing or treatment is required. PTCP was identified as being in vitro phenomenon which can be caused by following situation: EDTA-dependent platelet phenomena[7], platelet agglutination due to improper specimen collection, cold agglutination[8], platelet satellite phenomenon[9], large/ giant platelets[10], drug therapy[11]. The prevalence of PTCP in blood and platelet apheresis donors, with frequency ranging from $0.01-0.2 \%[12,13]$.PTCP was significantly higher in males aged 50 years or older [14]. PTCP caused by platelet satellitism and phagocytosis by neutrophils only occurs in approximately $0.1 \%$ in clinical $[15,16]$. Here, we present an extremely rare case of PTCP results from platelet phagocytosis by neutrophils, platelet lack of granules and giant platelet. EDTA-dependent autoantibodies triggers several signaling pathways that activate platelets, leading to their aggregation, satellitism or phagocytosis $[15,16]$.Platelet aggregation is the most common situation while platelet phagocytosis is the rarest one.Some studies suggest that platelet phagocytosis is related with EDTA-dependent autoantibodies, it usually occurs after platelet satellite phenomenon and is an extension of platelet satellite $[4,17]$. .n our case, we find platelet phagocytosis by neutrophils, platelet lack of granules and giant platelet in the patient's peripheral blood smear(Fig. 1). When the anticoagulant was changed from EDTA to sodium citrate, these phenomena still exist. At present, there are no reported cases of the simultaneous occurrence of these three abnormal forms. The optical platelet-counting method identifies platelets with laser light scatter technique shown as a reliable method for accurate platelet counting in thrombocytopenic patients, this technique combines scattered light and side fluorescence detectors., a fluorescent dye (oxazine) is used beforehand to stain platelets and reticulocytes[18-20].However, this technique also fails to detect engulfed platelets and platelets without granules In multiple tests, the number of platelets counted by manual was significantly higher than that counted by instrument (Table1). This also indicate that instrumentation is not a complete substitute for manual testing, and peripheral blood smears must be performed in cases of PTCP. The PTCP may have only minor pathophysiologic significance. However, this situation must be distinguished from true in vivo platelet clumps detected by chance during a blood test. As the in vitro phenomenon of PTCP could be misdiagnosed with thrombocytopenia, it does affect diagnostic, management, and therapeutic decisions

Although PTCP has been previously reported during therapy with abciximab, the incidence and significance of this occurrence are unknown, PTCP is a benign laboratory condition that does not increase bleeding, stroke, transfusion requirements or the need for repeat revascularization [21]. As one of the most effective chemotherapy medicines for tuberculosis (TB), rifampicin is widely used in China as there is high incidence of this disease. The common adverse effects of rifampicin are gastrointestinal disorders, skin rash, hepatotoxicity, etc, rifampicin is the most likely drug to cause thrombocytopenia in the course of antituberculous therapy [22,23], it can even cause disseminated intravascular coagulation (DIC) in patients [24].But, PTCP caused by antituberculous therapy has not been reported so far. In our 
case, before receiving anti-tuberculosis treatment, the patient showed no significant abnormality in the number $\left(101 \times 10^{9} / \mathrm{L}\right)$ and morphology of platelets. One month after antituberculous treatment (2HRZE/4HR), platelets count was significantly reduced by instrument detection ( $\left.56 \times 10^{9} / \mathrm{L}\right)$. However, through manual counting of peripheral blood smear, we found no significant change in the number of platelets in the patient $\left(136 \times 10^{9} / \mathrm{L}\right)$. During the course of treatment, this phenomenon persists (Table1), the patient did not show a tendency of bleeding or thrombosis, so we deduced that the patient developed PTCP. Three months after the end of treatment, no abnormal platelets were observed in the peripheral blood, so we speculated that the patient's PTCP was caused by anti-tuberculosis treatment. Of course, since we are reporting a single case, we have less evidence and didn't do any further research, our opinion is not conclusive considering that the mechanisms of drug-induced PTCP are extremely complex.

Here, we report it for the first time that an extremely rare case of PTCP results from platelet phagocytosis by neutrophils, platelet lack of granules and giant platelet. HRZE treatment may cause platelet morphology abnormal, resulting in PTCP. In such cases, we should regularly review the peripheral blood smear to ensure the accuracy of the results and avoid unnecessary examination and treatment. The emergence of PTCP may does not mean the presence of specific disorders.

\section{Declarations}

\section{Funding}

The present study was supported by National Natural Science Foundation of China (81602297).

\section{Competing interests}

The authors declare that they have no competinginterests.

\section{Data availability}

All data y are included in this article

\section{Code availability}

Not applicable

\section{Ethics approval}

Not applicable

\section{Author contributions}

$\mathrm{YZ}$ and JZ contribute to thesis selection and design, data collection; SL participate in data analysis and interpretation; FG contributes to critical review of the intellectual content of an article; YD and MZ contribute to the manuscript writing. 


\section{Consent to participate}

The study is supported by the patient's wife and she has signed informed consent.

Consent for publication

Not applicable

\section{References}

1. Chae H, Kim M, Lim J, Oh EJ, Kim Y, Han K: Novel method to dissociate platelet clumps in EDTAdependent pseudothrombocytopenia based on the pathophysiological mechanism. CLIN CHEM LAB MED 2012, 50(8):1387-1391.

2. García SJ, Merino JL, Rodríguez M, Velasco A, Moreno MC: [Pseudothrombocytopenia: incidence, causes and methods of detection]. Sangre (Barc) 1991, 36(3):197-200.

3. Zandecki M, Genevieve F, Gerard J, Godon A: Spurious counts and spurious results on haematology analysers: a review. Part l: platelets. INT J LAB HEMATOL 2007, 29(1):4-20.

4. Paul P, Kaur M, Kakkar N, Kumar N: Co-existent Platelet Phagocytosis, Satellitism and Clumping Causing Spurious Thrombocytopenia. Indian J Hematol Blood Transfus 2013, 29(3):158-160.

5. Criswell KA, Breider MA, Bleavins MR: EDTA-dependent platelet phagocytosis. A cytochemical, ultrastructural, and functional characterization. AM J CL IN PATHOL 2001, 115(3):376-384.

6. Holinstat M: Normal platelet function. Cancer Metastasis Rev 2017, 36(2):195-198.

7. Senzel L, Chang C: Platelet phagocytosis by neutrophils. BLOOD 2013, 122(9):1543.

8. Goyal P, Agrawal D, Kailash J, Singh S: Cold-induced pseudoneutropenia in human immunodeficiency virus infection: first case report and review of related articles. Indian J Hematol Blood Transfus 2014, 30(Suppl 1):148-150.

9. Sousa SM, Sousa TM, Silva CF, Mendes CC: Pseudothrombocytopenia: a case of platelet satellitism and phagocytosis by neutrophils. PLATELETS 2020, 31(4):541-543.

10. Noris P, Biino G, Pecci A, Civaschi E, Savoia A, Seri M, Melazzini F, Loffredo G, Russo G, Bozzi V et al: Platelet diameters in inherited thrombocytopenias: analysis of $\mathbf{3 7 6}$ patients with all known disorders. BLOOD 2014, 124(6):e4-e10.

11. Kam T, Alexander M: Drug-induced immune thrombocytopenia. J Pharm Pract 2014, 27(5):430-439.

12. Maslanka K, Marciniak-Bielak D, Szczepinski A: Pseudothrombocytopenia in blood donors. VOX SANG 2008, 95(4):349.

13. Sweeney JD, Holme S, Heaton WA, Campbell D, Bowen ML: Pseudothrombocytopenia in plateletpheresis donors. TRANSFUSION 1995, 35(1):46-49.

14. Xiao Y, Yu S, Xu Y: The Prevalence and Biochemical Profiles of EDTA-Dependent Pseudothrombocytopenia in a Generally Healthy Population. Acta Haemato/ 2015, 134(3):177-180. 
15. Lippi G, Plebani M: EDTA-dependent pseudothrombocytopenia: further insights and recommendations for prevention of a clinically threatening artifact. CLIN CHEM LAB MED 2012, 50(8):1281-1285.

16. Tan GC, Stalling M, Dennis G, Nunez M, Kahwash SB: Pseudothrombocytopenia due to Platelet Clumping: A Case Report and Brief Review of the Literature. Case Rep Hemato/ 2016, 2016:3036476.

17. Kovacs F, Varga M, Pataki Z, Rigo E: Pseudothrombocytopenia with multiple anticoagulant sample collection tubes. Interv Med App/ Sci 2016, 8(4):181-183.

18. Hummel K, Sachse M, Hoffmann J, van Dun L: Comparative evaluation of platelet counts in two hematology analyzers and potential effects on prophylactic platelet transfusion decisions. TRANSFUSION 2018, 58(10):2301-2308.

19. Sun Y, Hu Z, Huang Z, Chen H, Qin S, Jianing Z, Chen S, Qin X, Ye Y, Wang C: Compare the accuracy and precision of Coulter LH780, Mindray BC-6000 Plus, and Sysmex XN-9000 with the international reference flow cytometric method in platelet counting. PLOS ONE 2019, 14(5):e217298.

20. Kim HY, Bang SH, Cho D, Kim HJ, Kim SH: Performance evaluation of platelet counting of Abbott Alinity hq and Sysmex XN-9000 automated hematology analyzer compared with international reference method. INT J LAB HEMATOL 2021, 43(3):387-394.

21. Sane DC, Damaraju LV, Topol EJ, Cabot CF, Mascelli MA, Harrington RA, Simoons ML, Califf RM: Occurrence and clinical significance of pseudothrombocytopenia during abciximab therapy. $J A M$ COLL CARDIOL 2000, 36(1):75-83.

22. Arnold DM, Kukaswadia S, Nazi I, Esmail A, Dewar L, Smith JW, Warkentin TE, Kelton JG: A systematic evaluation of laboratory testing for drug-induced immune thrombocytopenia. J THROMB HAEMOST 2013, 11(1):169-176.

23. Vayne C, Guéry EA, Rollin J, Baglo T, Petermann R, Gruel Y: Pathophysiology and Diagnosis of DrugInduced Immune Thrombocytopenia. J CLIN MED 2020, 9(7).

24. Chen G, He JQ: Rifampicin-induced disseminated intravascular coagulation in pulmonary tuberculosis treatment: A case report and literature review. Medicine (Baltimore) 2017, 96(7):e6135.

\section{Figures}



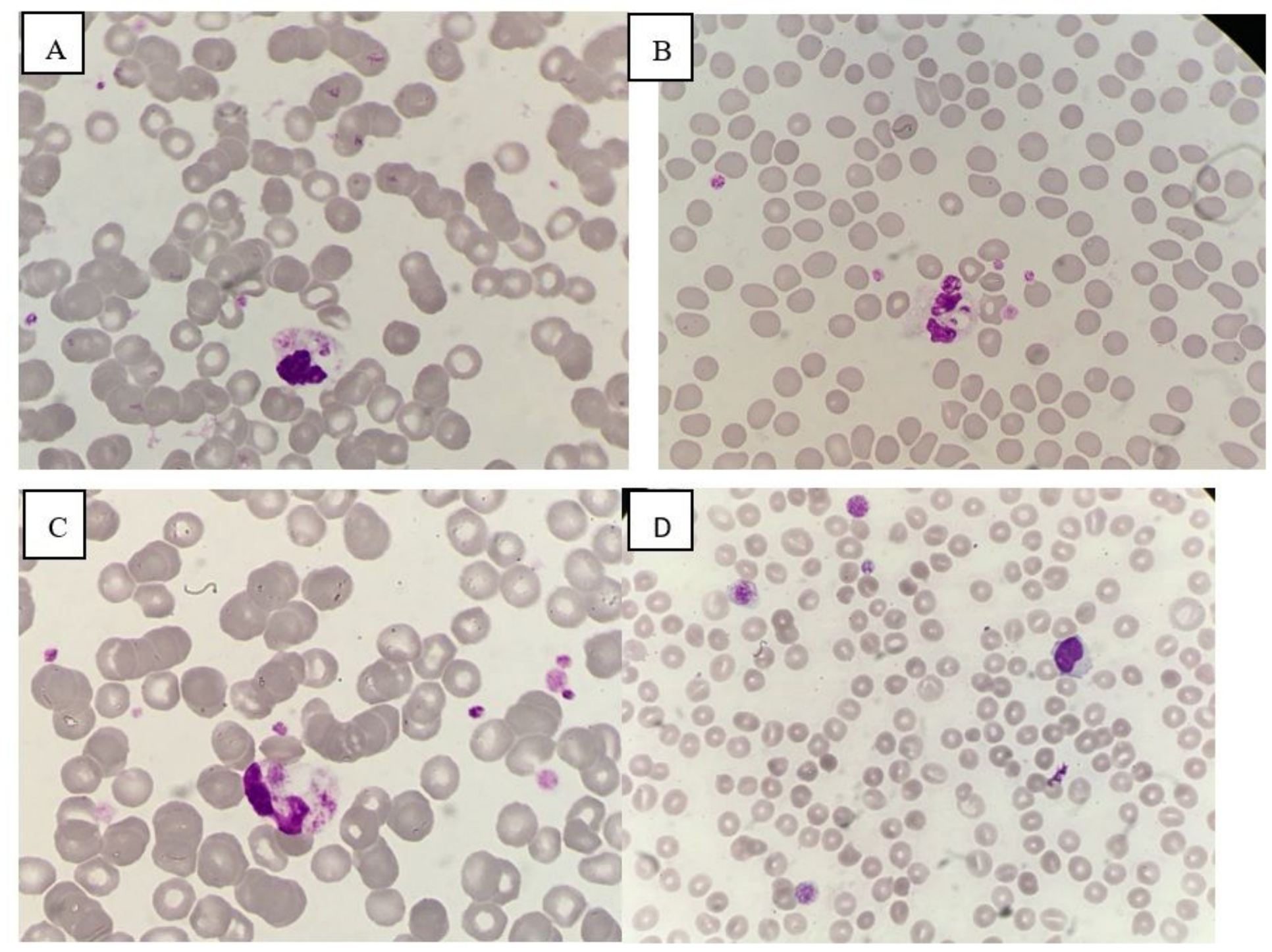

\section{Figure 1}

Wright-Giemsa-stained peripheral blood smear of the patient. Platelet phagocytosis by neutrophils, platelets lack of microparticle and giant platelets can be observed in the above pictures. 